\title{
Dynamic Balance Optimization Design of Spherical 4R Space Agency Based on UG
}

\author{
Zhou Kaihong ${ }^{1, a}$, Zhu Liang ${ }^{2, b}$ \\ ${ }^{1}$ Guilin University of Technology , Guilin, China \\ ${ }^{2}$ Guilin University of Technology , Guilin, China \\ a510429271@qq.com,b357266984@qq.com
}

Keywords: spherical 4R spatial mechanism; dynamic balance ; motion simulation; UG MOTION Abstract. Through 3D modeling and simulation software UG and the MOTION contained in it, the preliminary 3D modeling of 3D human vestibular training swivel chair which is the first generation of products based on spherical 4R spatial mechanism was completed, meanwhile the exact masses of counterbalance was obtained by optimization through an iteration method in UG and preliminary calculation, which realized the dynamic balance optimization design. And then we completed the motion simulation, the performance of the dynamic balance of the spherical 4R space agency had been greatly improved and verified by analyzing the data of motion simulation .

\section{Preface}

At present, the three-dimensional human vestibule training swivel chair is the first generation of products that produced which is based on the spherical $4 \mathrm{R}$ spatial mechanism. When the training swivel chair is in operation, the speed will be too fast or too slow suddenly because of the imbalance of the rod, and it will cause much vibration and noise, shorten the life of the machine, and even let the operator be in danger. Therefore, it is necessary to optimize the dynamic balancing of the spherical $4 \mathrm{R}$ spatial mechanism.

The spherical $4 \mathrm{R}$ spatial mechanism is a complicated spatial ${ }^{[1]}$ mechanism. In order to avoid the complex and abstract solving process in analytic method ${ }^{[2]}$ and kinematics analysis ${ }^{[3]}$, this paper makes use of UG's powerful modeling, assembly and mathematical functions to design its three-dimensional solid model. The weight of the counterbalance is obtained by the initial calculation, and then the optimal weight of the counterbalance is obtained by using the iterative method in UG. Finally, the motion simulation is used to verify the results in dynamic balance.

\section{D Modeling of Virtual Prototype of Spherical 4R Spatial Mechanism}

The spherical 4R spatial mechanism consists of 4 revolute pairs (A, B, C, D) intersecting the center of the sphere by the axis ${ }^{[4]}$.The organization diagram is shown in Figure 1. Utilize UG's powerful modeling and assembly functions to create a virtual prototype model by using optimized parts in the UG environment and set the corresponding quality parameters for each part, as shown in Figure 2.It will provide a good foundation for importing UG MOTION afterwards.

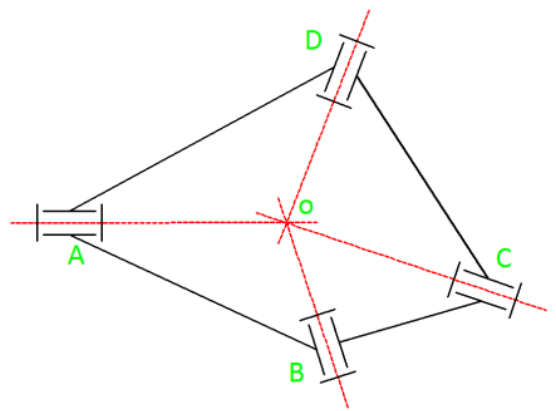

Fig. 1. Sketch of spherical $4 \mathrm{R}$ spatial mechanism 


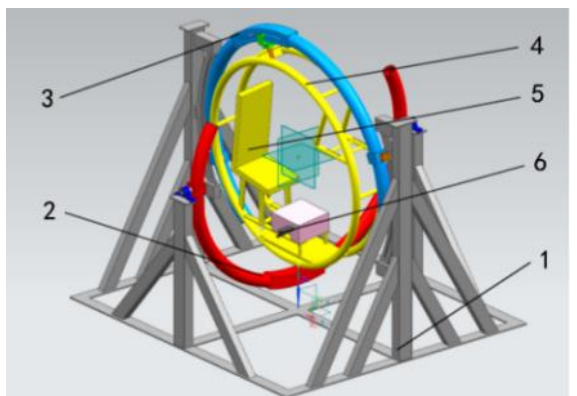

Fig. 2. 3D solid model of virtual prototype of spherical 4R spatial mechanism 1. base; 2 . first rocker; 3 . second rocker; 4 . seat bracket; 5 . seat; 6 . pedal shaft

\section{Dynamic Balance Analysis and Numerical Calculation}

In order to satisfy the static balance ${ }^{[5]}$, the center of mass of the system must be placed on the axis of rotation .It can ensure that the centrifugal force generated is zero during the rotation of the resultant force. To satisfy dynamic balance, it is necessary to ensure that the rotation of the centrifugal force caused by the torque is zero. The spherical $4 \mathrm{R}$ spatial mechanism is a complex spatial linkage mechanism, which consists of 4 revolute pairs. If you want to make the machine running smoothly, it is necessary to adjust the balance of the operation.

The preliminary analysis shows that the three-dimensional swivel chair mainly has two problems:the first rod and the second rod are unbalanced when rotating and the two shafts on the seat bracket are unbalanced when rotating.These forces cause unbalanced forces and moments, which need to be solved by adding a balance block, as shown in figure 3 . Then, the balance block mass $m_{3}$ and $m_{4}$ are added on the first rod and the second rod, and the human body simulation model is added on the seat.Assuming that the operator's weight $\mathrm{m}_{1}$ is $80 \mathrm{~kg}$.

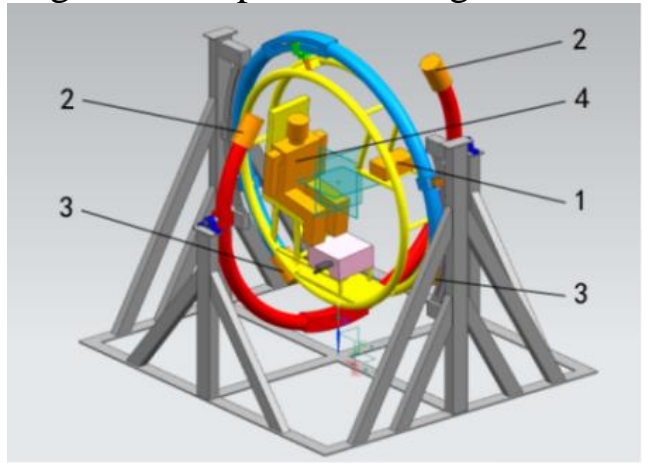

Fig. 3. 3D solid model of virtual prototype of spherical 4R spatial mechanism of counterweight

1. balance weight on the seat bracket $\mathrm{m}_{2} ; 2$. balance weight on the first $\operatorname{rod} \mathrm{m}_{3} ; 3$. balance weight on Dynamic balance calculation ${ }^{[6]}$ :

the second rod $\mathrm{m}_{4} ; 4$. weight of operator $\mathrm{m}_{1}$

$$
\begin{aligned}
& \sum P_{r}=\sum_{i=1}^{n}\left(m_{i} r_{i} \omega_{i}^{2}\right)=0 \\
& \sum M_{r}=\sum_{i=1}^{n}\left(L_{i} m_{i} r_{i} \omega_{i}^{2}\right)=0
\end{aligned}
$$

According to the actual parameters, the balance weight of $\mathrm{m}_{2}, \mathrm{~m}_{3}$ and $\mathrm{m}_{4}$ are $95.2 \mathrm{~kg}, 8.5 \mathrm{~kg}, 6.6 \mathrm{~kg}$ by the preliminary calculation.

To calculate the optimal weight of the balance block by using the iterative method in UG, you must let the center of mass is as close as possible to the center of the sphere $(0,0,0)$, and finally get the answer: the balance weight on the seat bracket $\mathrm{m}_{2}$ is $90.13 \mathrm{~kg}$, the center of mass coordinates is $(1.012,0.85 .-0.721)$; the first $\operatorname{rod} \mathrm{m}_{3}$ is $7.93 \mathrm{~kg}$, the center of mass coordinates is $(-0.204,0.460,0.000)$, and the second bar on the balance weight $\mathrm{m}_{4}$ is $7.63 \mathrm{~kg}$, the center of mass coordinates is $(-0.377,0.325,0.006)$. 


\section{Motion Simulation}

Enter the UG / Motion module, create a new simulation, and select the kinetic analysis type, create five links , 6 sports pairs. As shown in Figure 4, the five connecting rods are L001, L002, L003, L004, L005 respectively; six motion pairs are fixed sub-J001, rotating vice J002, J003, J004, J005, J006. Among them, J002 and J003 are fixed on the rack, J004 is the relative motion pair of connecting rod L002 and L004, J005 is the relative motion pair of connecting rod L002 and L003, and the J005 is coupled with J006.

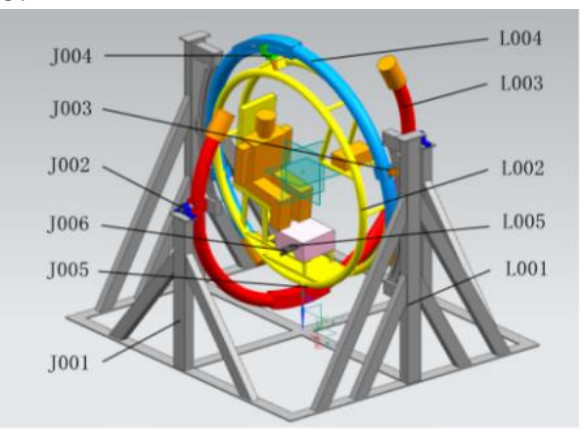

Fig. 4. Virtual prototype simulation model of spherical 4R spatial mechanism

Define motion driver. Select the rotation of J006, set the uniform speed of $180^{\circ} / \mathrm{s}$, select the human body mass A001 as the mark point. In the solution program, model check is to select the type of analysis for the kinematics / dynamics, duration of $2 \mathrm{~s}$, the number of steps for 360 .And then make a solution.

After the success of the solution, the whole or single step can be used to simulate the movement of animation or pictures. When the steps are 150, the simulation diagram is shown in Figure 5.

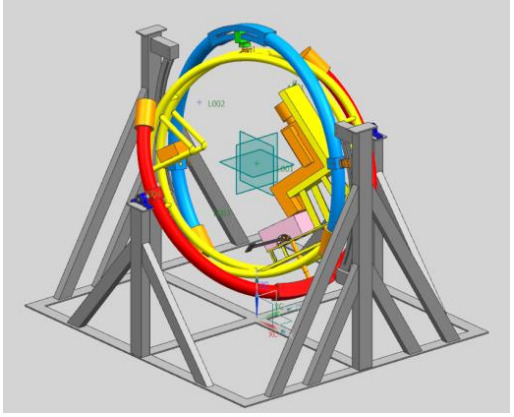

Fig. 5. Mechanism simulation diagram of the 150th step

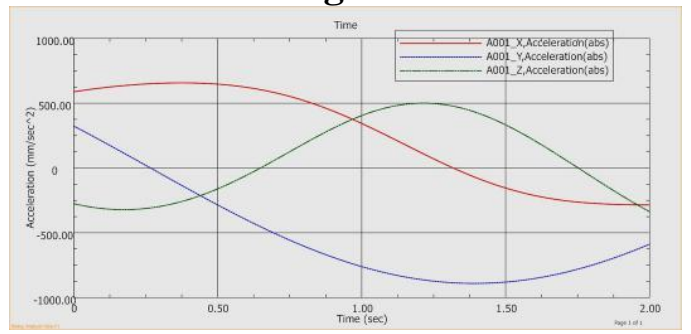

Fig. 6. Optimization of previous acceleration

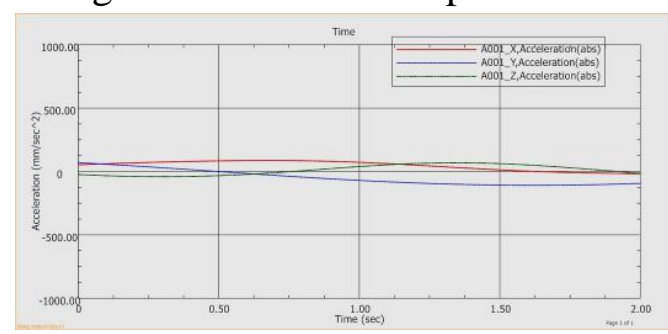

Fig. 7. Optimization of the acceleration

Call the Graphing function to draw the human body mass A001 marker point in the $\mathrm{x}, \mathrm{y}, \mathrm{z}$ three-axis upwards acceleration plot which is shown in figure 6, contrast with the optimization before observing the results. And optimization of previous acceleration is shown in figure 7.

Through analysis of the above two charts, we can see that the maximum value of $X$ axial acceleration is reduced from $660 \mathrm{~mm} / \mathrm{s}^{2}$ to $88 \mathrm{~mm} / \mathrm{s}^{2}$, and the axial acceleration of $\mathrm{Y}$ decreases from $892 \mathrm{~mm} / \mathrm{s}^{2}$ to $95 \mathrm{~mm} / \mathrm{s}^{2}$, and the axial acceleration of $\mathrm{Z}$ decreases from $500 \mathrm{~mm} / \mathrm{s}^{2}$ to $45 \mathrm{~mm} / \mathrm{s}^{2}$.The $X$ axis of unbalanced force is reduced from $52.8 \mathrm{~N}$ to near 0 , the $\mathrm{Y}$ axis of unbalanced force is reduced from $72 \mathrm{~N}$ to near 0 , the $\mathrm{Z}$ axis of unbalanced force is reduced from $40 \mathrm{~N}$ to near 0 . Therefore, people affected by the unbalanced force is greatly reduced, the unbalance torque is also significantly reduced accordingly. Therefore, the effect of dynamic balance is optimized, which solves the problem that the speed is accelerated or too slow suddenly during the running process. 


\section{Concluding Remarks}

In this paper, UG and UG MOTION are used to design the three-dimensional solid model. The weight of counterbalance is obtained by the initial calculation, and then the optimal balance block is obtained by using the iterative method in UG. The simulation results show that the unbalanced force is significantly reduced and the dynamic balance effect is improved, which indicates that the optimization design method is practicable. In order to let the center of gravity is as close as possible to the center of the sphere, the structure of seat bracket needs to be improved, the seat position and the installation of the balance block position need to be designed reasonably.

\section{References}

[1] In Chinese. Wu Qiong, Yan Min, Wang Wenbo. Position analysis and motion synthesis of spherical 4R mechanism[J]. Journal of Beijing Institute Of Fashion Technology, 1998,02:67 71.

[2] In Chinese. Zhou Jianming, Xu Shijie, Peng Xiaofang, Hu Qinglong, Zeng Zhaohai. Wobble yoke drive mechanism dynamic balance optimization design of [J]. diesel engine, 2009: thirty-first volume third.

[3] In Chinese. Chen Youqing.3- (2SPS) three translational and online static equilibrium study [D]. Jiangsu Nanjing: Nanjing University of Science and Technology,2011,03.

[4] In Chinese. Wang Guangming, Shi Lixin. Spherical 4R mechanism type diagram method four position synthesis of the [J]. Chinese mechanical engineering,2013,03:336 340.

[5] In Chinese. Bai Shuanggang, Hu Mengquan, Duan Jintan. Static equilibrium analysis of six degrees of freedom for ship borne catapult launch [J].Journal of Air Force Engineering University (NATURAL SCIENCE EDITION), volume, volume third.

[6] D.Chablat, J.Angeles.The computation of all 4R serial spherical wrists with an isotropicarchitecture, ASME J.Mech. Design 125 (2003) 275 280. 